\title{
Phase-averaged mean properties of turbulent flow developing around a fluttering sheet of net
}

\author{
Elkhadim Bouhoubeiny $^{\mathrm{a}, \mathrm{b}}$, Philippe Druault ${ }^{\mathrm{b}, \mathrm{c}, *}$, Grégory Germain ${ }^{\mathrm{a}}$
}

\author{
a IFREMER, Marine Structures Laboratory, 150 Quai Gambetta, 62321 Boulogne-sur-Mer, France \\ b Université Pierre et Marie Curie-Paris 6, UMR 7190, Institut Jean Le Rond d'Alembert, 75252 Paris cedex 5- \\ France \\ ${ }^{\mathrm{c}}$ CNRS, UMR 7190, Institut Jean Le Rond d'Alembert, 75252 Paris cedex 5, France \\ *: Corresponding author : Philippe Druault, email address : philippe.druault@upmc.fr
}

\begin{abstract}
:
Time-Resolved Particle Image Velocimetry (PIV) measurements are performed to investigate the hydrodynamic flow interaction with a representative piece of fishing net structure. Using the advanced post-processing mathematical tool based on Proper Orthogonal Decomposition, the phase averaged mean properties of turbulent flows are extracted from PIV database, demonstrating the existence of a Turbulent Boundary Layer (TBL) flow developing all around the sheet of net. The results also show that the mesh opening and so the turbulent flow passing through the structure are strongly influenced by the structure oscillations. This work allows us to demonstrate that the drag computation of fishing gear, commonly based on uniform velocity, needs to consider not only the local velocity deficit near the horizontal part of the net but also the instantaneous oscillations of the sheet of net.
\end{abstract}

\section{Highlights}

We provide a better understanding of the flow through netting structures. We show the development of Turbulent Boundary Flow around horizontal net structure. We demonstrate the great influence of fluttering net structure onto hydrodynamics. Such study will help to improve the fishing gears drag computation.

Keywords: Fishing net structure ; Hydrodynamics ; PIV ; Turbulent boundary layer flow

\section{Introduction}

The mesh fishing structures and their flow interactions play an important role in the fishing vessel energy efficiency due to the impact of the drag force on fuel consumption (Germain et al., 2010). Commonly, computations of drag force have been carried out by using empirical formulations using the towing velocity as a reference water velocity (Priour, 2009), assumed that water flow inside the net is equal to the corresponding towing speed, even if it is well admitted that the flow speed inside the cod-end is smaller than the one outside the cod-end and also than the towing speed. These small velocity differences may lead to some modifications in local drag 
force estimations. The fishing structure drag force determination remains today complex due to the fluid/structure interactions that induces large deformation and oscillations of the net and modifies instantaneously the local drag force $[3,4]$.

It is also now clear that turbulent flow developing inside cod-end structure has a significant impact on fish behaviour and then on selectivity. Engaas et al. [5] showed that a modification of water flow in the trawl cod-end leads to improve the fish escapement. They also showed that the trawl geometry has a non-negligible effect on selectivity. This can be related to the work of O'Neill et al. [6] who illustrated the effect of cod-end pulsing on selectivity. Previous analyses mainly based on one point measurement $[7,8]$ shown the effect of turbulence on maneuvering of fish swimming and also on potential use of active stimulating device. Very recently, Time Resolved PIV (Particle Image Velocimetry) measurements have been carried out to investigate the flow around an oscillating cod-end model [9]. It has been emphasized the turbulent flow interaction with the fluterring cod-end structure. Nevertheless, the analysis of the turbulent flow inside a cod-end is quite complex. In a realistic flow configuration, a full investigation of the 3D turbulent flow interacting with the fishing net structure is not possible not only numerically but also experimentally.

We propose in this study to experimentally investigate the turbulent flow interacting with a representative piece of fishing net structure, even if such structure does not correspond to any real fishing gear. However, such preliminary study will permit to elucidate the hydrodynamic behavior of such a simplified structure which is not fully understood and for which accurate predictions still remain today difficult. It is then expected that present results would have several implications in a more realistic flow configurations. Present analysis related to a simplified piece of fishing net structure allows the investigation of different turbulent flows developing around an arbitrarily oscillating fishing net structure: 1) the boundary layer developing around the horizontal part and 2) the turbulent flow passing through the inclined one. Based on advanced post-processing tool based on Proper Orthogonal Decomposition (POD) [10, 11], the phase averaged mean properties of these flows are examined as a function of multiple parameters such as structure oscillations and porosity.

After the description of the experimental facility in section 2, preliminary analyses of instantaneous velocity measurements are presented and the POD phase-averaged operator is briefly detailed. In section 3, POD analysis is detailed. Then in sections 4 and 5, TBL flows and turbulent flow passing the curved part of the fishing structure are successively analyzed as a function of structure motion. 


\section{Description of PIV measurements and post- processing tools}

\section{$2.1 \quad$ Experimental set-up}

Experiments are carried out in the IFREMER (French Institute for the Exploitation of the Sea) wave and current circulation flume tank [12, 9]. A rectangular sheet of net of $3 \mathrm{~m}$ length by $2 \mathrm{~m}$ width (120 meshes length by 80 meshes width) is used. The sheet of net is made of polyamide twine of $2.2 \mathrm{~mm}$ diameter and diamond-shaped meshes $l=22 \mathrm{~mm}$ (the length of the meshes between two knots). It is maintained in the tank only by two ropes, so the structure is free to move in the water flow (Figure 1-top). In order to obtain a shape having incidence changes encountered on real trawl, the sheet of net is mounted on a rectangular rope canvas like shown in Fig 1 (bottom). Even if the free downstream end of the sheet of net plays a dominant role in the oscillatory behavior of the structure, the structure motion is mainly two-dimensional with a maximum vertical amplitude less than $10 \mathrm{~cm}$. The $3 D$ deformation of the sheet of net leads mainly to a $2 D$ motion of the net knots in the vertical measurement plane (symmetrical axis of the structure).

An input streamwise velocity of $U_{\infty}=0.85 \mathrm{~m} \cdot \mathrm{s}^{-1}$ is imposed with a turbulence intensity rate $(T I)$ of $3 \%$, with:

$$
T I=\frac{\sqrt{\frac{1}{3}\left(\overline{u^{\prime 2}}+\overline{v^{\prime 2}}+\overline{w^{\prime 2}}\right)}}{U_{\infty}}
$$

where an overbar indicates the classical Reynolds time average and the fluctuating parts of the velocity field are indicated with a prime index. Note that the time average transverse and spanwise velocity component are null. The corresponding data have been measured with a 2D Laser Doppler Velocimetry system in two steps to acquire the three velocity components, in the far field where the flow is uniform.

The corresponding Reynolds number based on the diamond-shaped meshes and the upstream velocity $U_{\infty}$ is given by:

$$
R e_{\infty}=\frac{U_{\infty} l}{\nu}
$$

where $\nu$ denotes the fluid kinematic viscosity, $\nu \approx 10^{-6} \mathrm{~m}^{2} \cdot \mathrm{s}^{-1}$. The studied Reynolds number is then 17600 .

\subsection{PIV measurement system}

Two-dimensional PIV system is used to determine the streamwise $u$ and transversal $v$ velocity components along respectively the $x$ and $z$ directions. Seeding particles (silver-plated glass spheres of $15 \mu \mathrm{m}$ diameter) are introduced in the 


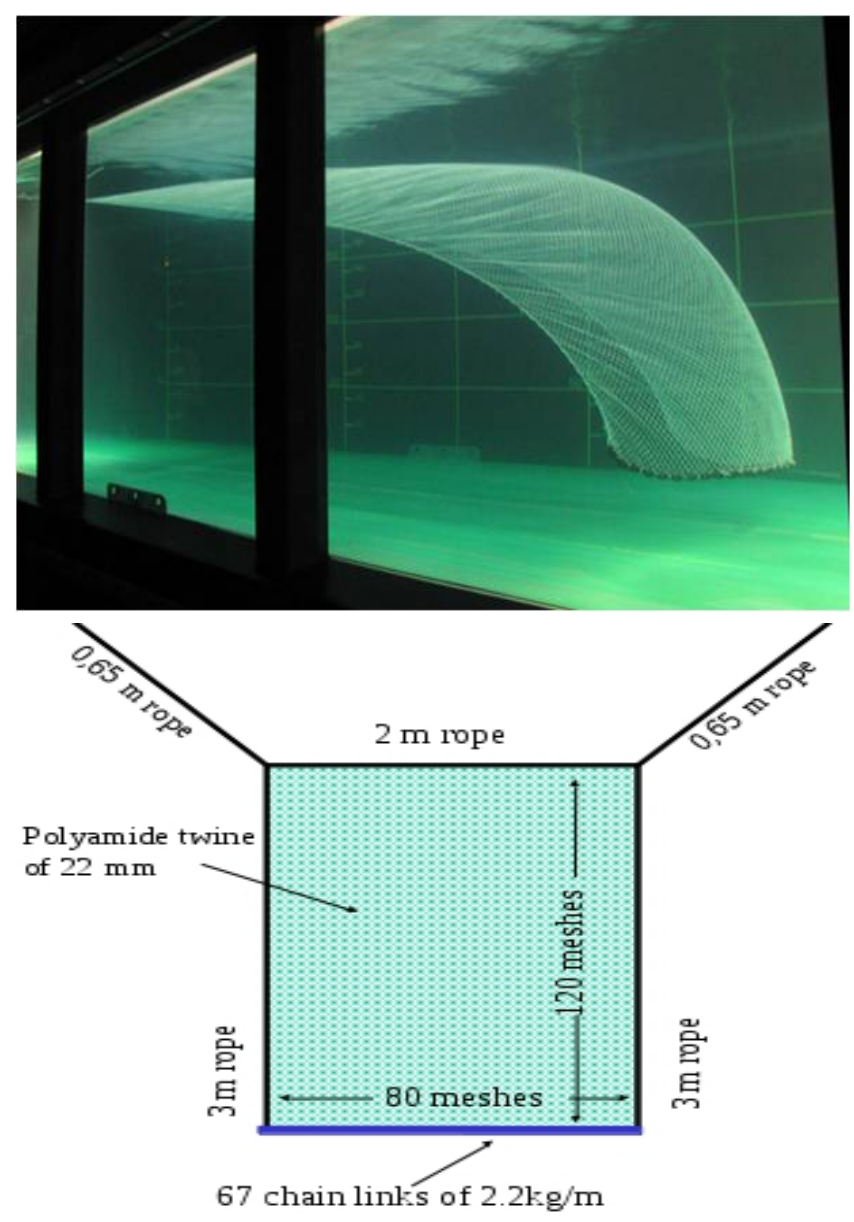

Figure 1: Top: Sheet of net located in the flume tank during trials at $0.85 \mathrm{~m} / \mathrm{s}$. Bottom: Description of the sheet of net assembly.

water and illuminated by a standard, frequency-doubled, double-cavity Nd:YAG laser (NewWave, Gemini PIV) with a pulse energy of up to $120 \mathrm{~mJ}$ per pulse at $532 \mathrm{~nm}$, and maximum repetition of $15 \mathrm{~Hz}$. A CCD camera with a $60 \mathrm{~mm}$ focal lens coupled to a PC running image acquisition software is used to acquire images. The physical area of approximately $0.38 \mathrm{~m}$ by $0.28 \mathrm{~m}$ is imaged onto 1600 by 1200 pixels $^{2}$. The final interrogation window size is $16 \times 16$ pixels $^{2}$ with a $50 \%$ overlap. Then, an interpolation based on the $3 \times 3$ neighbors replaces false vectors. In each instantaneous PIV image, $n_{x} \times n_{z}=199 \times 149$ instantaneous velocity vectors are finally provided with a spatial resolution of $1.9 \mathrm{~mm}$ in both directions. Details about PIV system and its associated post-processing algorithms can be found in [13].

PIV measurements are carried out in several 2D planes shown in Figure 2. 


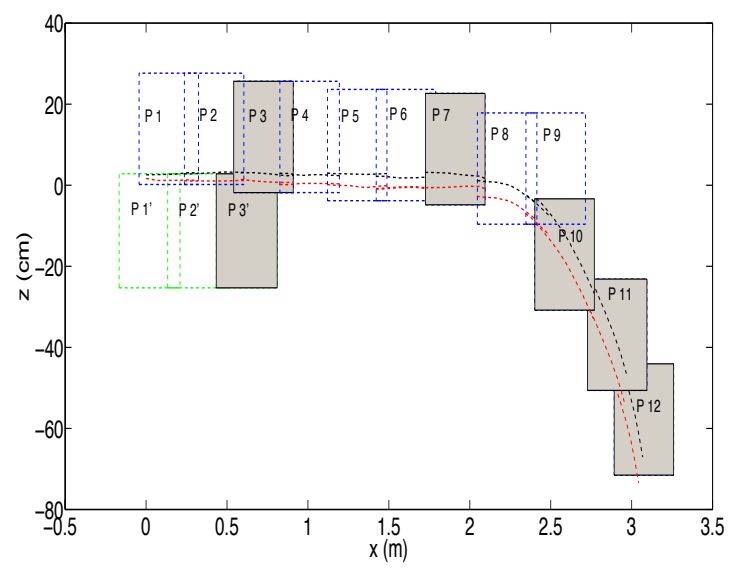

Figure 2: PIV measurement planes with the highest (black dashed line) and lowest (red dashed line) locations of the fluterring structure.

These overlapped planes are located in the vertical median plane of the sheet of net. Note that along the horizontal part of the mesh structure (from plane 1 to plane 7 ), no edge effects are observable in the vertical plane under investigation [13]. The first 7 planes permit to access the TBL development on the upper surface (blue dotted line in figure 2). Planes $P 1^{\prime}$ to $P 3^{\prime}$ are considered to determine the TBL development in the inner surface of the sheet of net (green dotted line in figure 2). Flow passing inclined part of the sheet of net are measured in planes $P 8$ to $P 12$. In each measurement plane, 818 instantaneous velocity fields are obtained, and the time separation between two PIV measurements is $0.25 \mathrm{~s}$ that corresponds to an acquisition frequency of $4 \mathrm{~Hz}$.

Due to the flexibility and the small thickness of the structure, a motion tracking technique allowing the measurement of the instantaneous motion of the deformable fishing net during PIV measurements is used. This technique detailed in [14] allows to track the motion of reference points $(0.8 \mathrm{~cm}$ diameter solid balls) visible in each PIV frame. Note that the targets are not in the main symmetrical axis of the piece of net in order to not disturb the flow measurement made in parallel. The introduced gap is more or less half a length mesh (approximately $2 \mathrm{~cm}$ ). Then, in each PIV plane (from $P 1$ to $P 12$ ), instantaneous velocity vectors located under the detected sheet of net interface during measurements are put to zero value. In a similar way, for PIV planes $P 1^{\prime}$ to $P 3^{\prime}$, velocity field located over the surface are also put to zero value. Note that the implementation of such tracking technique is valid because the structure motion is only in the 2D PIV plane, no spanwise motion is really observed. 


\subsection{First analysis: Instantaneous PIV velocity fields}

As a first investigation, selected instantaneous velocity fields are regarded. Figure 3 displays two instantaneous PIV measurements of the streamwise velocity component, obtained in plane measurement $P 3$. These two fields are obtained at two different instants that correspond to the highest and the lowest positions of the sheet of net during measurements. Instantaneous velocity fields are normalized with the upstream reference velocity $U_{\infty}=0.85 \mathrm{~m} \cdot \mathrm{s}^{-1}$. Such instantaneous representation clearly indicates the spatial development of the TBL flow around the net parts at low angle of incidence not only above the sheet of net but also inside (same behaviour for plan $P 1$ to $P 7$ and $P^{\prime} 1$ to $P^{\prime} 3$ ). These instantaneous pictures also emphasize that the streamwise velocity component changes according to the position of the structure. It is then confirmed that the TBL flow is function of the random motion of the structure [10]. When the mesh structure rises to its highest amplitude it seems that the spatial extent of the outer TBL is greater than when the structure is at its lowest position. Conversely, when regarding the inner TBL development (above the net structure, plane $P^{\prime} 3$ ), it is observed that the spatial extent of the inner TBL increases when the fishing structure rises its lowest position.
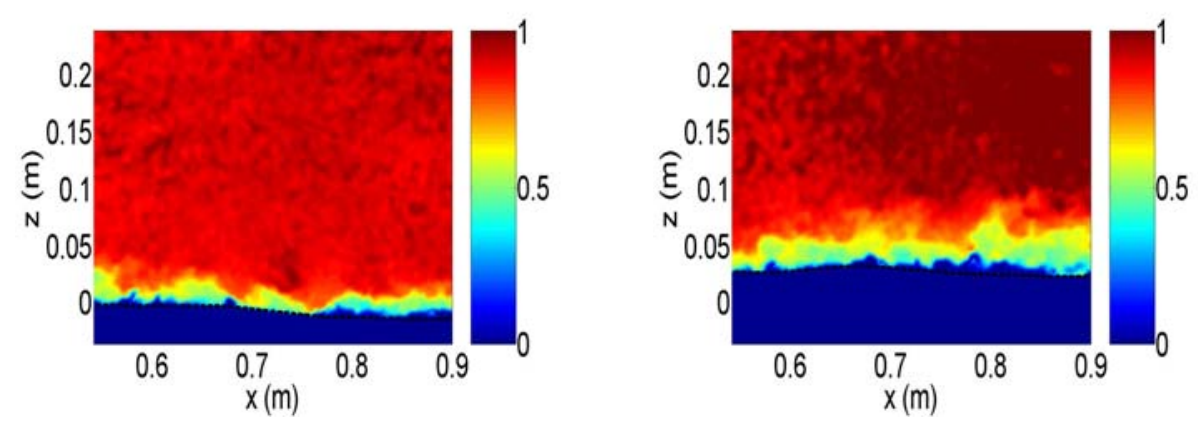

Figure 3: Instantaneous streamwise velocity component (normalized with $U_{\infty}$ ) in plane 3 at the lowest (left) and highest (right) position of the structure.

Figure 4 represents two normalized instantaneous streamwise velocity components associated with the highest and lowest positions of the sheet of net in plane $P 11$, showing that the flow passing inclined sheet of net is function of the motion of the structure. Indeed, when moving structure rises its crest (highest amplitude), it seems that the mass flow is higher than the one observed when moving structure is at its lowest position.

These preliminary results show the influence of the structure motion on the turbulent flow (TBL and flow passing inclined structure) pointing the necessity to use specific analysis tools, that is done in sections 4 and 5 for the phase averaged mean properties characterization of both flows thanks to a Proper Orthogonal Decomposition (POD) analysis. 

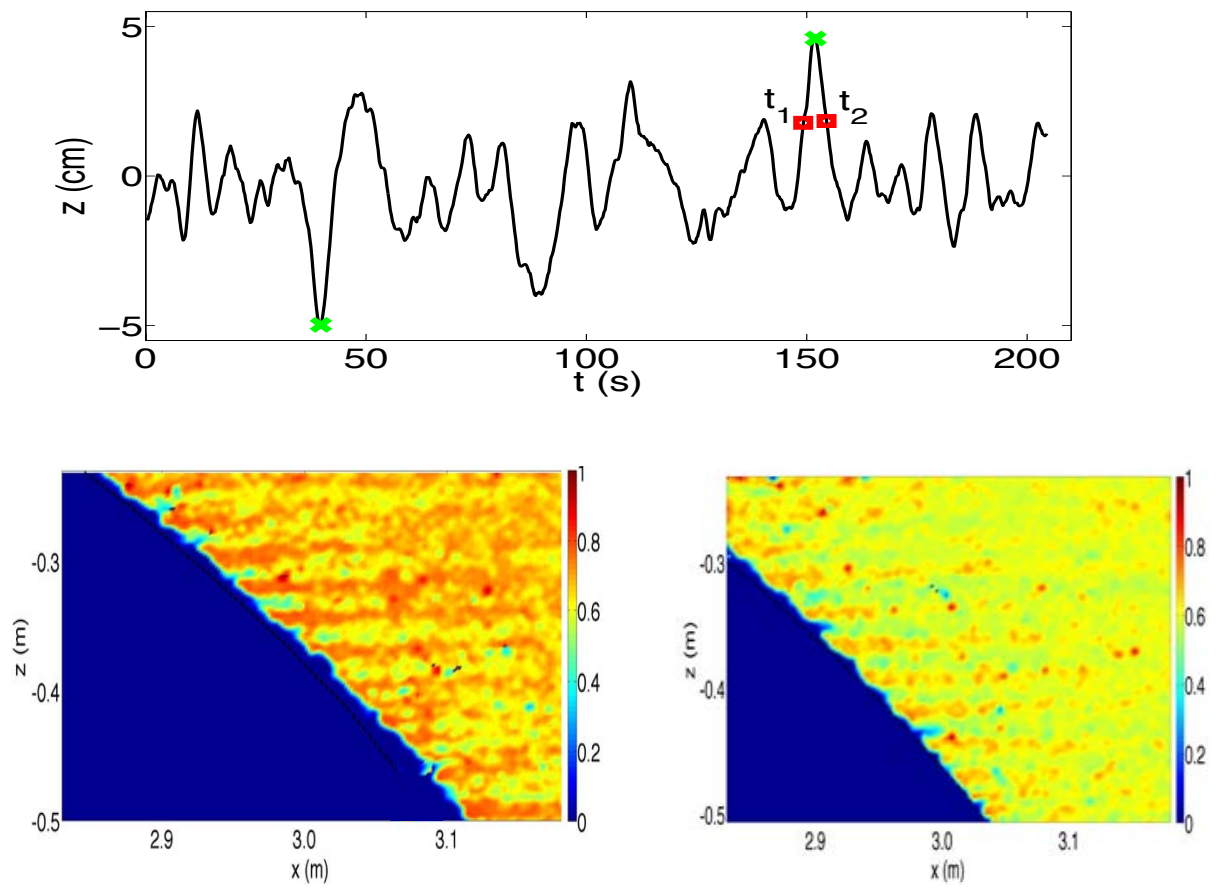

Figure 4: Time evolution of the transverse motion of the sheet of net in Plane 11 (top) and instantaneous streamwise velocity component (normalized with $U_{\infty}$ ) obtained at the highest (left) and lowest (right) position of the structure. These instants are indicated with green crosses in the top graph. Instants $t_{1}$ and $t_{2}$ are related to figure 9 .

\subsection{Phase averaged operation}

Due to the random motion of the sheet of net and the limited number of available instantaneous PIV samples, classical post-processing mathematical tools can not be applied to easily determine the phase average mean flow properties. When dealing with fully unsteady flow, the aim is to decompose instantaneously each velocity field $\vec{V}(x, z, t)$ as follows:

$$
\mathbf{V}(x, z, t)=\mathbf{V}_{\mathbf{m}}(x, z, t)+\mathbf{v}^{\prime}(x, z, t)
$$

where $\mathbf{V}_{\mathbf{m}}$ and $\mathbf{v}^{\prime}$ correspond respectively to the phase averaged mean velocity field and its associated fluctuating part. Then, based on previous works [10], POD technique can be used to approach the mean phase averaged flow field at each instant. 


\subsubsection{Proper Orthogonal Decomposition}

POD procedure is a mathematical technique allowing the decomposition of a random flow variable according to its energy content [15, 16]. The first POD modes capture the most part of the kinetic energy of the flow with a minimum of terms, compared to any flow decomposition. That is explained the great development of POD application to turbulent flows due to its efficiency in extracting the large scale coherent structures present in turbulent flow [17, 18, 19]. Briefly, orthogonal spatial POD modes named $\Phi$ are computed from the Fredholm integral [16]. They correspond to eigenfunctions of the kernel of the integral problem that is the two point correlation tensor of available database. PIV

velocity database, $\mathbf{V}(x, z, t)$, is generally decomposed thanks to the summation of uncorrelated POD modes as follows:

$$
\mathbf{V}(x, z, t)=\sum_{n=1}^{N_{\text {mod }}} a^{(n)}(t) \boldsymbol{\Phi}^{(n)}(x, z)
$$

where $a^{(n)}(t)$ satisfying $a^{(n)}(t) a^{(m)}(t)=\delta_{n m}$ ( $\delta$ is the kronecker symbol), are the projection of instantaneous velocity field onto the $n^{t h}$ POD mode.

\subsubsection{POD phase averaged operator}

When dealing with fully unsteady flow field like for instance flow around arbitrarily moving mesh interface, POD can not be performed from the fluctuating flow field as usual. Nevertheless, exploiting the POD energy efficiency, Druault et al. [10] proposed to carry out POD to the fully unsteady available 2D PIV database. Knowing that the mean energetic feature in such a case is the mean flow field, they demonstrated that the first two POD modes are well associated with the instantaneous phase averaged mean flow field:

$$
u_{m}(x, z, t)=\sum_{n=1}^{2} a^{(n)}(t) \Phi_{u}^{(n)}(x, z)
$$

where $u_{m}$ is the phase averaged mean streamwise velocity component. Such POD operator technique has been applied with success from PIV measurements performed around the horizontal part of the modeled bottom trawl. More details of this phase averaged technique can be found in [10].

The purpose of this work is then to extent such methodology to other PIV database allowing the characterization of TBL development all around the fluttering fishing structure and also of the turbulent flow passing inclined fluttering sheet of net. Such application will permit to elucidate the effect of structure motion on the hydrodynamic flow developing all around the sheet of net.

\section{POD analysis}

In this section, POD is successively performed from each 2D PIV database related to each individual plane. Note that scalar and vector POD decompo- 


\begin{tabular}{|c|c|c|c|c|}
\hline & Plane $P 3$ & Plane $P^{\prime} 3$ & Plane $P 7$ & Plane $P 11$ \\
\hline $1^{\text {st }}$ mode & 99.3 & 99.11 & 98.09 & 95.17 \\
\hline $2^{\text {nd }}$ mode & 0.31 & 0.28 & 0.59 & 1.97 \\
\hline $3^{\text {rd }}$ mode & 0.04 & 0.04 & 0.14 & 0.62 \\
\hline
\end{tabular}

Table 1: Energy content of the first 3 POD modes expressed in percentage value.

sitions are successively performed. No real differences are observed between both POD applications, especially in the first POD modes content [13], justifying the presentation of only scalar POD results in the following. In fact, for present convective flows under investigation, the transverse velocity component is always several orders of magnitude smaller than the one of the streamwise component. Then, scalar PODs are applied to each raw PIV database where $N_{t}=818$ instantaneous streamwise velocity components are available. In each plane, that provides $N_{\text {mod }}=N_{t}$ POD modes.

\subsection{Analysis of POD energy content}

The POD energy content is investigated by regarding $\sum_{n=1}^{N} \lambda^{(n)} / \sum_{n=1}^{N_{\text {mod }}} \lambda^{(n)}$ as a function of $N$. It provides an estimation of mode energy content relative to the total energy of the streamwise component. Table 1 indicates the energy content of the first 3 POD modes in Planes $P 3, P 3^{\prime}, P 7$ and $P 11$. The high energy content of POD mode 1 is directly linked to the fact that the entire (not only the fluctuating part) streamwise component is treated in the POD application. When regarding results of PIV measurement planes $P 3$ and $P^{\prime} 3$, quasi similar POD energy convergences are observed. Moreover, as expected as far as the flow becomes more and more turbulent, the first POD mode represents less and less energy. This is especially true for plane measurement $P 11$ results, where the flow complexity related to the fluid passage through the fluttering sheet of net is clearly indicated.

\subsection{Analysis of spatial POD modes}

The topologies of the first 2 POD modes computed from two PIV databases (Planes $P^{\prime} 3$ and $P 11$ ) are presented in figure 5 . In each graph, the highest and lowest positions of the sheet of net during measurements are included as black dotted lines. POD modes obtained from other PIV databases (from Planes $P 1$ to $P 7$ and from Planes $P^{\prime} 1$ to $P^{\prime} 2$ ) are similar to the ones for Plane $P^{\prime} 3$. For each PIV database, the first 2 POD modes are of higher amplitude than the one of POD mode remainder. The color contours of the first mode show the topology of a time averaged field calculated from instantaneous velocity fields without taking into account the sheet of net. This first POD mode can be viewed as the signature of a global mean flow but which does not represent any realistic mean flow field due to the unsteady character of the present turbulent flow. Furthermore, the area swept by the motion of the structure is well recovered 
thanks to the second POD mode. The last mode can be considered as a marker of the structure displacement during PIV measurements.
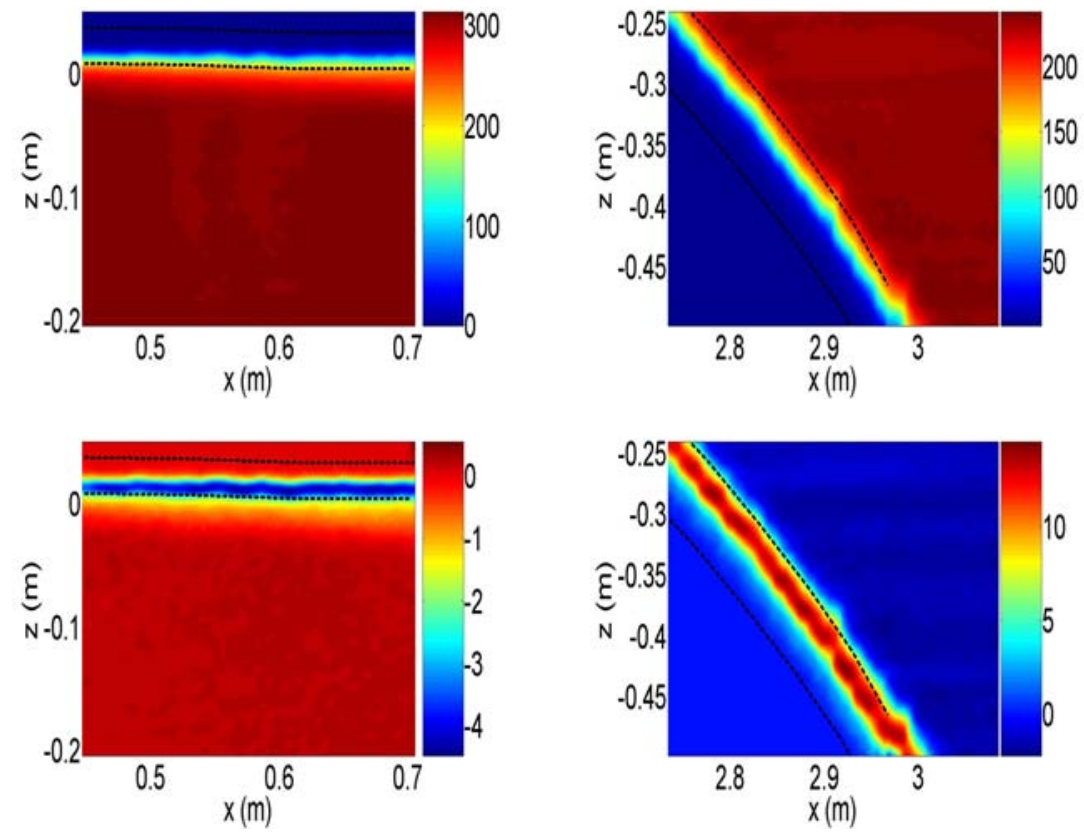

Figure 5: Isosurface of the first 2 POD modes (mode 1 on top and mode 2 on bottom), for Plane $P^{\prime} 3$ (left) and $P 11$ (right). Black dotted lines indicate the highest and lowest positions of the sheet of net.

\subsection{Analysis of temporal POD modes}

The time evolutions of the first two POD temporal coefficients are presented in figure 6. They are superimposed onto the time evolution of transverse oscillations measured thanks to the motion tracking technique. The first POD mode remains always quasi-monotonic and constant in each case. Conversely, the magnitude of the second POD coefficient is higher than the one related to the first POD mode. Moreover, such representation emphasizes that in each case the time evolution of the second POD coefficient is quite in phase with the time evolution of the sheet of net during PIV measurement.

From these results, whatever the fully unsteady turbulent flow under investigation, the following conclusions can be drawn: i) the first POD mode extracts favorably the global time averaged flow field without taking into account the fluttering interface; ii) the second mode follows exactly the temporal dynamic of random motion of the fluttering structure. These conclusions are similar for each flow configuration (net parts at low and high incidence with the flow) and 

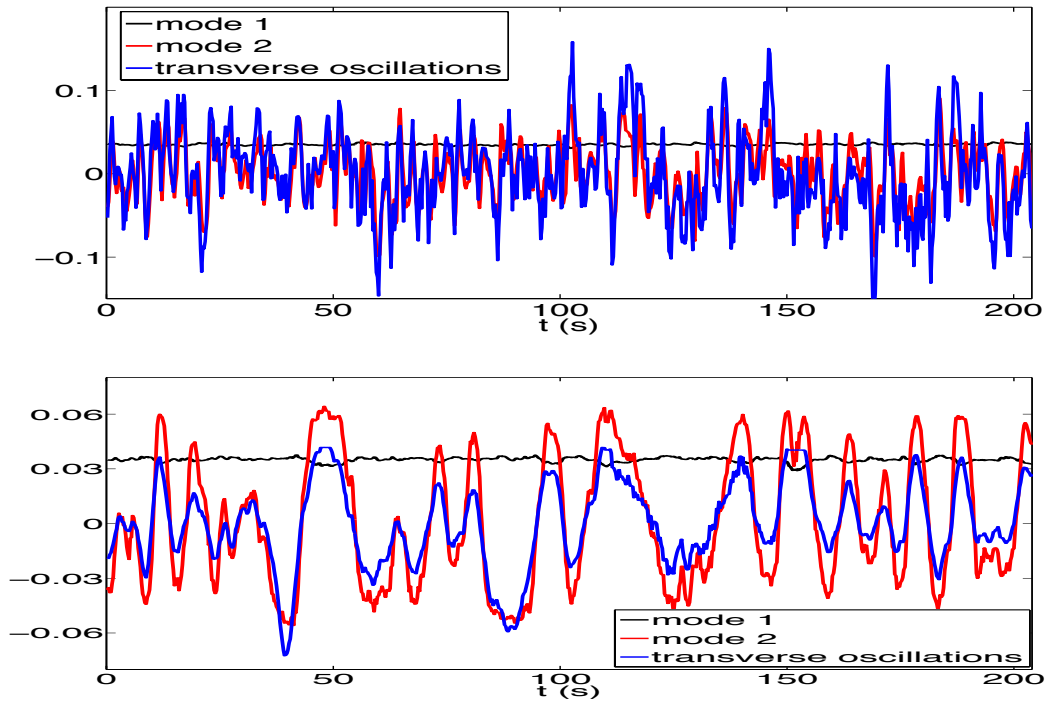

Figure 6: Time evolution of the first two POD coefficients in plan 3 (top) and 11 (bottom). The time evolutions of transverse oscillations in each measurement plane are also represented for comparison.

confirm previous work [10] that POD acts as a phase averaging operator for unsteady turbulent flow. A linear combination of the first 2 POD modes enables then to identify the phase averaged mean flow field in connection to the motion of the fluterring sheet of net. Therefore, in each PIV measurement plane, the phase averaged mean streamwise component is determined as follows:

$$
u_{m}(x, z, t)=\sum_{n=1}^{2} a^{(n)}(t) \phi^{(n)}(x, z)
$$

\section{Turbulent boundary layer developing inner and outer the structure}

The phase averaged mean streamwise velocity component is computed thanks to equation 6 . As an illustration, figure 7 displays selected phase averaged velocity profiles of $u_{m}(x, z, t)$ associated with selected instants in planes $P 3$ and $P 7$. These instants correspond to 1) the one related to the highest position of the structure during measurements and 2) during an upward motion of the structure. These results indicate that the TBL flow developing all around the fishing net structure is function of the structure motion.

To pursue the investigation of the TBL development as a function of structure motion, figure 8 represents the phase averaged mean flow field computed in each measurement plane at similar instants. For instance, three instants are 
retained: the first one corresponds to the highest position reached by the sheet of net in each individually measurement plane, the second and third ones correspond to the median position of the sheet of net but during an upward and downward structure motion respectively. It is confirmed that when the sheet of net reaches its highest position, the thickness of the TBL flow is higher than in the other position. Furthermore, an upstream motion of the structure promotes the extent of the TBL flow.

Based on the knowledge on the phase averaged mean flow field, the TBL thicknesses of $\delta_{90}(x, t)$ are computed at each $x$-position for each time step $t$. This boundary layer thickness associated with the local position of the structure is defined as the value of $z$ for which $u_{m}(x, z, t)=0.9 U_{\infty}$. Figure 8 (bottom-right) displays the instantaneous evolution of $\delta_{90}$ along the upper part of the sheet of net. Even if the spatial development of TBL flow is clearly visible for both parts of the sheet of net, $\delta_{90}(x, t)$ greatly varies as a function of the random motion of this structure that locally also modifies the incidence and the porosity of the fishing structure. These results are in agreement with previous ones dealing with the analysis of the TBL flow developing over a fluttering modeled bottom trawl [10].
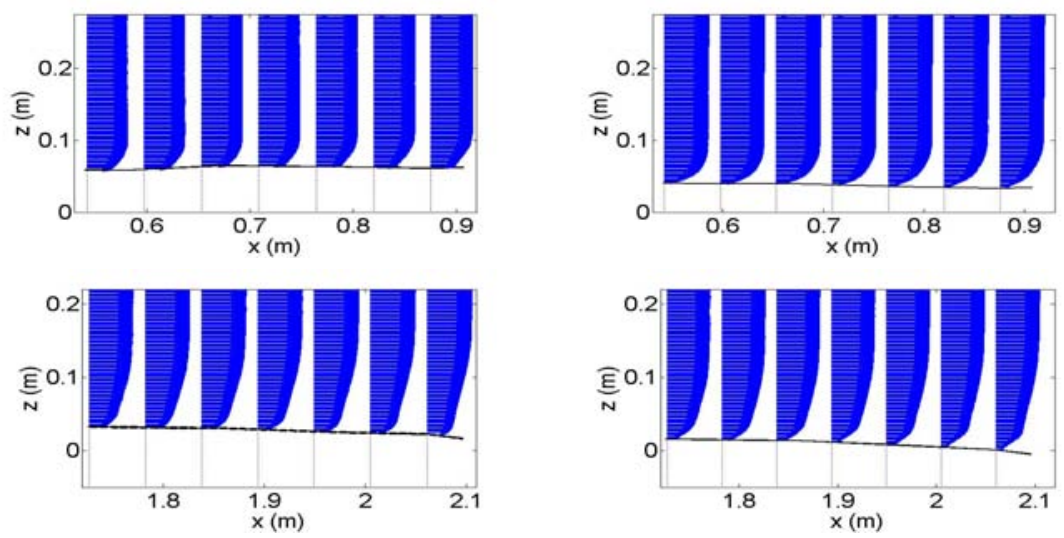

Figure 7: Examples of phase averaged mean velocity profiles in the planes $P 3$ (top) and $P 7$ (bottom) for the highest (left hand side) and median position of the structure (right hand side).

When regarding the time evolutions of $\delta_{90}$ thickness and of transverse oscillations one observes that the development of TBL flow is in phase with the transverse oscillations for the TBL flow developing over the fishing structure. More precisely, $\delta_{90}(t)$ related to TBL flow developing over the sheet of net increases during an upward movement of the sheet of net. Conversely, when analyzing the evolution of $\delta_{90}(t)$ associated with the inner development of TBL flow, an opposite behavior is observed. During a downward motion of the structure, the TBL flow grows more favorably than during an upward motion of the structure. 
a)

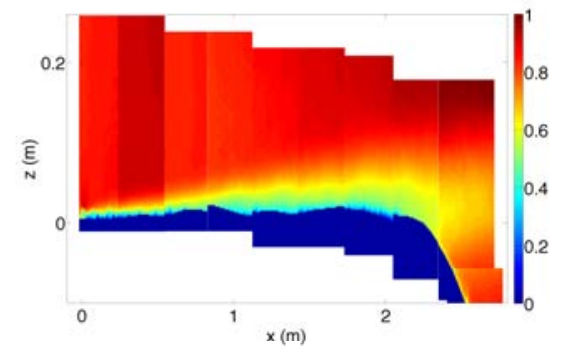

c)

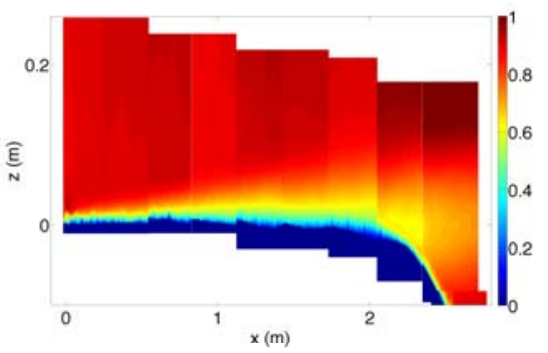

b)

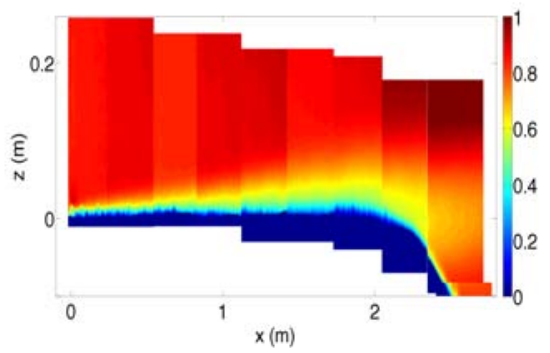

d)

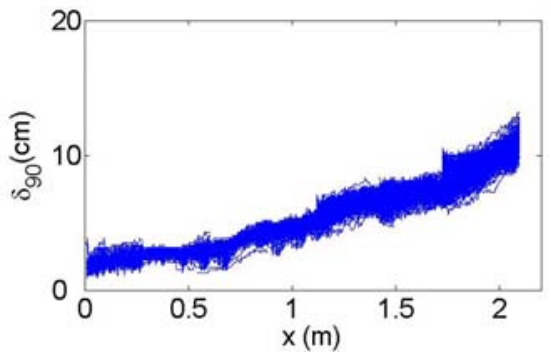

Figure 8: Phase averaged mean velocity around the rectangular sheet of net at three instants that correspond in each plane to a) the crest amplitude of structure oscillations; b) a median position during an downward structure movement; c) a median position during an upward structure movement. d) Spatial evolution of the instantaneous value of boundary layer thickness along the sheet of net.

\section{Phase averaged mean properties of turbulent flow passing through inclined part of the net}

Following POD analysis, the POD averaged operator is implemented from PIV database related to measurement planes around inclined structure. The phase averaged mean streamwise velocity component is then obtained by projected instantaneous flow field onto the first two POD modes (see equation 6). As a first investigation, figure 9 represents this phase averaged mean velocity field normalized with $U_{\infty}$, and obtained at two different instants, $t_{1}$ and $t_{2}$ in each inclined measurement plane. These instants correspond to the same position for the fluttering sheet of net but during an upward and downward movement respectively (see for instance figure 4 for $t_{1}$ and $t_{2}$ determination related to Plane P11). At these instants the sheet of net has the same incidence with the flow. First, the phase averaged mean flow velocity field is strongly reduced downstream the sheet of net confirming previous investigations [20]. Second, the influence of the structure motion on fluid passage is clearly indicated (see figure 9). Indeed, when the structure goes upward the flow passes more favorably 
through the sheet of net.
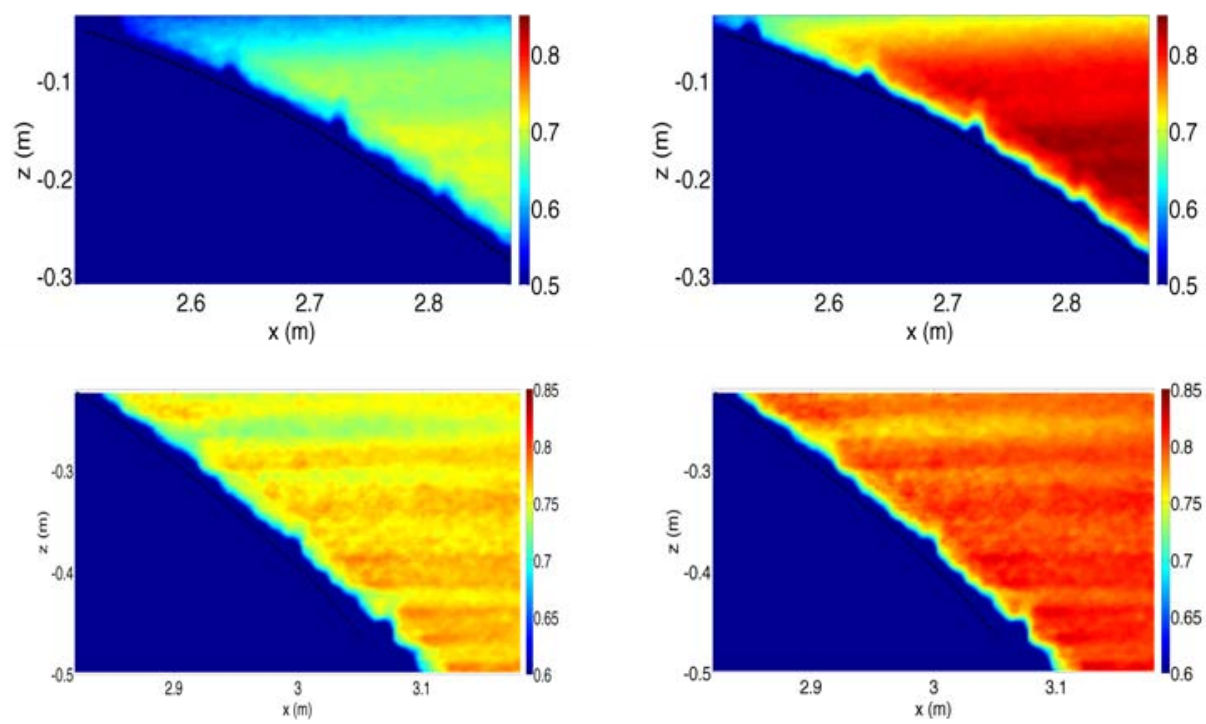

Figure 9: Phase averaged mean streamwise component obtained at two instants associated with an upward (on the right) and downward (on the left) motion of the structure in plane $P 10$ (top) and $P 11$ (bottom).

To confirm such behavior, the phase averaged streamwise components $u_{m}\left(\mathbf{X}_{i}, t\right)$ are extracted at fixed points $\left(\mathbf{X}_{P 10}, \mathbf{X}_{P 11}, \mathbf{X}_{P 12}\right)=((2.7,-0.12),(2.95,-0.34),(3.12,-0.64))$ located near the mesh moving structure in each measurement plane $P 10, P 11$ and $P 12$ respectively. When computing the time average of each signal $u_{m}\left(\mathbf{X}_{i}, t\right)$, one obtains $\overline{u_{m}\left(\mathbf{X}_{P 10}\right)}<\overline{u_{m}\left(\mathbf{X}_{P 11}\right)}<\overline{u_{m}\left(\mathbf{X}_{P 12}\right)}$, where an overline indicates the time average. This is directly related to the inclination of the sheet of net. Indeed, the angle with the flow increases with the measurement plane number (see figure 1-right). Globally, similar behaviors are observed in each plane. Figure 10 on top displays the time evolution of $u_{m}\left(\mathbf{X}_{P 10}, t\right)$. As each time representation is superimposed onto the transverse oscillations of the sheet of net, it is confirmed that the structure motion greatly influences the fluid passage through the structure. As a matter of fact, in each plane, when the structure goes upward, the phase averaged flow amplitude decreases. Conversely, when the structure moves downward, the flow passes more favorably through the structure. The amplitude of the flow variabilities due to the structure motion are non-negligible. In certain cases, these variations represent around $20 \%$ of the reference flow velocity $U_{\infty}$.

To further investigate the velocity reduction as a function of transverse oscillations, the mesh opening is now regarding. Note that the porosity can not be properly defined thanks to an analysis of only 2D PIV images. Indeed, the porosity is classically defined thanks to the knowledge of the variations of the 
mesh opening area [21] which is not available in present work. One then proposes to focus on the mesh opening that can be obtained from 2D PIV database. Thus, a measure of the porosity can be approached by estimating the length and the angle of two consecutive balls located on the fishing net structure. For such an investigation, the tracking system implemented to determine instantaneously the structure motion is considered. By examining the time evolution of the length between two consecutive balls, a measure of the mesh opening is possible. Figure 10 (middle) presents such time evolution which is superimposed onto the time evolution of the transverse oscillations. As expected, both signals are opposite phase. When the structure goes upward, the length between two consecutive balls decreases and allows the mesh opening. In contrast, when the structure goes downward, the length increases reducing the mesh opening and then the fluid passage. Another important parameter is the angle between the

flow and the sheet of net. Thanks to the determination of the instantaneous ball motion, the time evolution of angle between two consecutive balls is then accessed. Figure 10 (bottom) presents such time evolution which is also superimposed onto the time evolution of the transverse oscillations. We also retrieve that both signals are opposite phase. However, note that the angle variations during measurements are very small (of several degrees) avoiding any real effect on the fluid passage through the structure. Finally, the structure motion has a great influence on the mesh opening and then certainly on the porosity. Even if the transverse oscillations are of amplitude of several centimeters, one observes some great change in the local porosity and then in the fluid passage and fluid exchange.

\section{Conclusions}

This study first shown that the POD method can be used as a phase averaging operator allowing to characterize both types of flow encountered over this sheet of net: the development of boundary layer around the parts at low incidence with the flow and the flow passing through the inclined sheet of net at high incidence.

Concerning the TBL flow investigation, POD applications allows the determination of the phase averaged mean flow properties of TBL flow developing all around the fluttering mesh structures. Knowing that a realistic trawl is composed partly of a great number of horizontal panels, it seems then necessary to take into account the local deficit of velocity near horizontal part to improve the entire drag computation of the fishing gear. Consequently, we believe that incorporating more realistic experimental velocity database in associated drag model would be beneficial for the drag computation of fishing net structure. That certainly leads to greatly modify locally the drag coefficient which may vary significantly with respect to the horizontal extent of the trawl. Moreover, present results show the influence of structure motion onto hydrodynamics. As stated in the introduction part, the drag computation is also function of structure oscillations. Based on such experimental database, it would be possible 

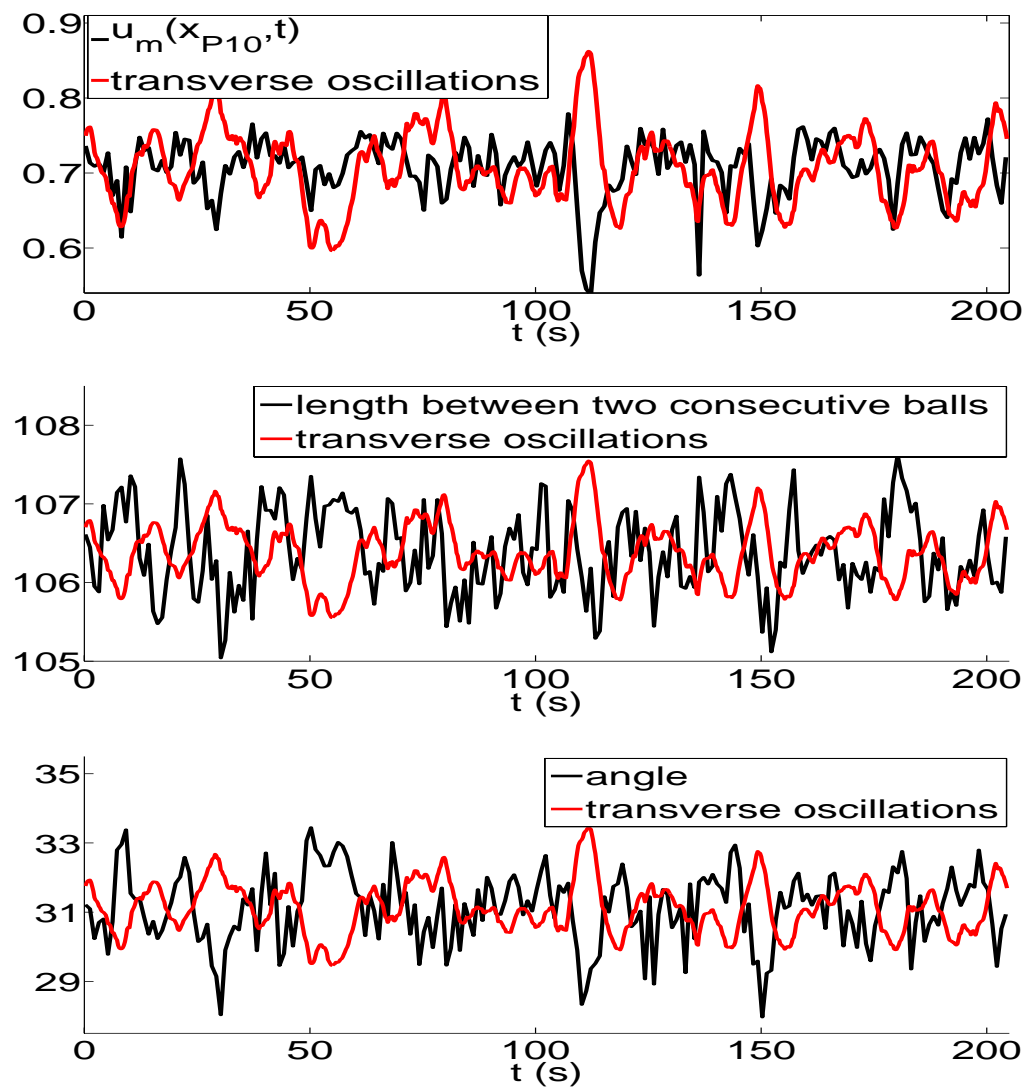

Figure 10: Time evolution of a/ the phase averaged streamwise velocity component $u_{m}\left(\mathbf{X}_{P 10}, t\right)$ extracted at a fixed point $\mathbf{X}_{P 10}=(2.7,-0.12)$ (top), b/ the length (middle) and angle (bottom) between two consecutive balls during PIV measurements in Plane $P 10$. 
to estimate the variations of local velocity and then of drag computation as a function of structure oscillations.

Concerning the flow passing through inclined structures, it is confirmed that velocity field is strongly reduced downstream the sheet of net [20]. In this sense, by studying the flow passing through inclined structures, one also emphasizes the great influence of structure motion on the mass flow and then on fluid exchange. It is observed that transverse oscillations lead to greatly modify the mesh opening. A upward motion of the structure generates mesh opening and then leads to increase the flow passage. Such observation may have implications in the physiology and behavior of fish which are greatly influenced by spatial and temporal fluctuations of selected parameters [22].

\section{References}

\section{References}

[1] G. Germain, P. Druault, R. Lewandowski, B. Vincent, J. Billard, Hydropêche: a way to improve energy efficiency of fishing devices, in: 1st Int. Symp. on Fishing Vessel Energy efficiency, 2010.

[2] D. Priour, Numerical optimisation of trawls design to improve their energy efficiency, Fisheries Research 98 (2009) 40-50.

[3] Z. Zheng, N. Zhang, Frequency effects on lift and drag for flow past an oscillating cylinder, J. Fluids Struct. 24 (2008) 382-399.

[4] L. Liu, T. Kinoshita, R. Wan, W. Bao, H. Itakura, Experimental investigation and analysis of hydrodynamic characteristics of a net panel oscillating in water, Ocean Eng. 47 (2012) 19-29.

[5] A. Engaas, D. Foster, B. Hataway, J. Watson, I. Workman, The behavioural response of juvenile red snapper (lutjanus campechanus) to shrimp trawls that utilise water flow modifications to induce escapement, Mar. Tech. Soc. J. 33 (1999) 43-50.

[6] F. O'Neill, S. McKay, J. Ward, A. Strickland, R. Kynoch, A. Zuur, An investigation of the relationship between sea state induced vessel motion and cod-end selection, Fisheries Research 60 (2003) 107-130.

[7] Y. Kim, Analysis of turbulence and tilt by in-situ measurements inside the cod-end of a shrimp beam trawl, Ocean Eng. 53 (2012) 6-15.

[8] Y. Kim, Analysis of the turbulent flow and tilt in the cod-end of a bottom trawl during fishing operations, Ocean Eng. 64 (2013) 100-108.

[9] E. Bouhoubeiny, G. Germain, P. Druault, Time-resolved PIV investigations of the flow field around rigid cod-end net structure, Fisheries Research 108 (2-3) (2011) 344-355. 
[10] P. Druault, E. Bouhoubeiny, G. Germain, POD investigation of the unsteady turbulent boundary layer developing over porous moving flexible fishing net structure, Exp. Fluids 53 (1) (2012) 277-292.

[11] J. Lumley, The structure of inhomogeneous turbulent flows, in: Yaglom, Tatarsky (Eds.), Atm. Turb. and Radio wave Prop., 1967, pp. 166-178.

[12] G. Pichot, G. Germain, D. Priour, On the experimental study of the flow around a fishing net, Eur. J. Mech. B/Fluids (2009) 103-116.

[13] E. Bouhoubeiny, Caractérisation de l'écoulement autour de structures souples et poreuses : Application aux engins de pêche, Ph.D. thesis, University Pierre and Marie Curie - Paris 6 (2012).

[14] P. Druault, G. Germain, J. Facq, PIV measurements combined with the motion tracking technique to analyze flow a around moving porous structure, J. Fluids Struct. (In revision).

[15] L. Sirovich, Turbulence and the dynamics of coherent structures. part I: Coherent structures, Q. Appl. Math XLV (1987) 561-571.

[16] P. Holmes, J. Lumley, G. Berkooz, Turbulence, coherent structures, dynamical systems and symmetry., Cambridge monograph on mechanics eds., 1996.

[17] P. Druault, P. Guibert, F. Alizon, Use of Proper Orthogonal Decomposition for time interpolation from PIV data, Exp. Fluids 39 (2005) 1009-1023.

[18] S. Roudnitzky, P. Druault, P. Guibert, Proper Orthogonal Decomposition of in-cylinder engine flow into mean component, coherent structures and random Gaussian fluctuations, J. of Turbulence 7 (70) (2006) 1-19.

[19] P. Druault, C. Chaillou, Use of Proper Orthogonal Decomposition for reconstructing the 3D in-cylinder mean-flow field from PIV data, C.R. Mecanique 335 (1) (2007) 42-47.

[20] D. Johansson, J. Juell, F. Oppedal, J. Stiansen, K. Ruohonen, The influence of the pycnocline and cage resistance on current flow, oxygen flux and swimming behaviour of atlantic salmon (salmo salar l) in production cages., Aquaculture 265 (1) (2007) 271-285.

[21] S. Gjosund, B. Enerhaug, Flow through nets and trawls of low porosity, Ocean Eng. 37 (2010) 345-354.

[22] P. Klebert, P. Lader, L. Gansel, F. Oppedal, Hydrodynamic interactions on net panel and aquaculture fish cages: A review, Ocean Eng. 58 (2013) 260-274. 